\section{Klebsiella pneumoniae and acute anterior uveitis in ankylosing spondylitis}

Acute anterior uveitis (AAU) is frequently associated with ankylosing spondylitis (AS). It is probably not a complication of AS as it may often manifest before the onset of the disease. Acute anterior uveitis also occurs in Reiter's disease, ulcerative colitis, and Crohn's disease. When sacroiliitis is present, all of these conditions are strongly associated with the HLA B27 antigen. ${ }^{1}$ The incidence of HLA B27 in all individuals presenting to eye clinics with AAU is $58^{\circ}{ }_{0} 0^{2}$ Although infective agents are known to cause some forms of AAU, most cases are idiopathic or associated with diseases whose aetiology is unknown. We have reported evidence of a cross-reactivity among three Gramnegative micro-organisms-Enterobacter aerogenes, Klebsiella pneumoniae, and Yersinia entercolitica-and HLA B27 positive lymphocytes, ${ }^{3}$ and have also found that $K l$ pneumoniae was found more frequently in AS patients with active disease than in controls or patients with inactive disease. ${ }^{4}$ We now report, in detail, our findings relating to AAU in our group of patients with AS.

\section{Patients, methods, and results}

We have examined 190 patients with AS on 765 separate occasions. All patients fulfilled the New York criteria for AS. ${ }^{5}$ Patients with early or possible ankylosing spondylitis were excluded from this study. Of the 155 patients tissue typed, 139 were positive for HLA B27 $(90 \%)$. All patients with AAU were seen, the diagnosis confirmed, and treatment instituted by their ophthalmic physician. Faecal culture studies were carried out as described. ${ }^{4}$ All episodes where patients or controls were taking antibiotics were excluded from the study. A total of 159 healthy hospital-associated controls provided one faecal sample each, and 93 patients with rheumatoid arthritis regularly attending a research clinic provided faecal samples on 250 visits. The interval between each visit was a minimum of three months. Fifty female physiotherapy students, on their first day of contact with a hospital environment, also provided single faecal samples.

The results are shown in the table. Three patients had two episodes of

Comparison of positive and negative faecal cultures for Klebsiella/Enterobacter spp in AS patients with and without $A A U$, hospital controls, rheumatoid arthritis outpatients, and female physiotherapy students

\begin{tabular}{|c|c|c|c|c|c|}
\hline & \multicolumn{2}{|c|}{ AS patients } & \multirow{2}{*}{$\begin{array}{l}\text { Hospital } \\
\text { controls }\end{array}$} & \multirow{2}{*}{$\underset{\text { outpatients }}{\mathrm{RA}}$} & \multirow{2}{*}{$\begin{array}{l}\text { Physiotherapy } \\
\text { students }\end{array}$} \\
\hline & $\begin{array}{l}\text { With } \\
\text { AAU }\end{array}$ & $\begin{array}{l}\text { Without } \\
\text { AAU }\end{array}$ & & & \\
\hline $\begin{array}{l}\text { Positive cultures } \\
\text { Negative cultures }\end{array}$ & $\begin{array}{r}13 \\
4\end{array}$ & $\begin{array}{l}229 \\
536\end{array}$ & $\begin{array}{r}51 \\
106\end{array}$ & $\begin{array}{r}49 \\
201\end{array}$ & $\begin{array}{r}5 \\
45\end{array}$ \\
\hline
\end{tabular}

$\chi^{2}$ between AS patients with and without $\mathrm{AAU}=16.86(\mathrm{P}<0.001)$

AAU during the study and each was counted separately. Positive cultures for Kl pneumoniae were found in 13 out of 17 episodes $(76 \%)$. Klebsiella ! Enterobacter spp were identified on 229 out of 763 occasions $(30 \%)$, at which AAU was not found. The incidence in hospital controls, outpatients with rheumatoid arthritis, and female physiotherapy students was $33 \%$, $20 \%$, and $10 \%$ respectively. Many of the patients with AAU had positive cultures for klebsiella for some months before the development of AAU as well as at the time of assessment. Two cases were excluded because no cultures were available at the time of the AAU episode but previous cultures taken 9 and 12 weeks before gave positive results. One other case was excluded because no culture was available at the onset of AAU, although the culture result was positive when tested four weeks later and mild signs still present.

\section{Comment}

The cause of the joint and eye inflammation in HLA B27 positive individuals is unknown. An infective agent has been suspected, particularly in view of the evidence that reactive arthritis can occur after acute dysentery with salmonella, shigella, or Yersinia enterocolitica in HLA B27 positive individuals. Kl pneumoniae is only conditionally pathogenic and is commonly found in the gastrointestinal tract. Our finding that this organism is frequently associated with and often precedes AAU supports the hypothesis that many of the acute inflammatory episodes in HLA B27 positive patients whether acute peripheral arthritis, spinal disease, or eye inflammation are all responses to a common aetiological trigger. Whether this trigger is Kl pneumoniae remains to be seen.

Requests for reprints to RE.

${ }^{1}$ Brewerton, D A, Arthritis and Rheumatism, 1976, 19, 656.

2 Brewerton, D A, Annals of Rheumatic Diseases, 1975, 3, suppl, p 33.

${ }^{3}$ Ebringer, A, et al, in HLA and Disease, ed J Dausset and A Svejgaard, p 27. INSERM, Paris, 1976.

${ }^{4}$ Ebringer, R W, et al, Annals of the Rheumatic Diseases, 1978, 37, 146.

${ }^{5}$ Bennet, P H, and Wood, P H N, in Population Studies of the Rheumatic Diseases, ed P H Bennet and P H N Wood, p 456. Amsterdam, Excerpta Medica Foundation, 1968.

(Accepted 29 December 1978)

Departments of Rheumatology and Microbiology, Middlesex Hospital, London W1P 9PG

ROLAND EBRINGER, MRCP, Matilda Poole Fellow in rheumatology DIANE CAWDELL, AIMLT, technician

Immunology Unit, Department of Biochemistry and Microbiology, Queen Elizabeth College, London

ALAN EBRINGER, MD, FRACP, senior lecturer

\section{Prenatal treatment of fetal thyrotoxicosis}

Fetal thyrotoxicosis may be predicted and diagnosed but there are few reported attempts to treat it before delivery. ${ }^{1-3}$

\section{Case report}

A 27-year-old woman presented 11 weeks advanced in her second pregnancy. At 24 years she had had a subtotal thyroidectomy for Graves's disease. Pretibial myxoedema developed within one year of the operation. During her first pregnancy at the age of 25 she was euthyroid. At 33 weeks' gestation she delivered spontaneously a boy of $1.83 \mathrm{~kg}$ (25th centile for gestational age). At birth this child was severely thyrotoxic, with a free thyroxine index (FTI) of 37.5 (reference range $3 \cdot 2-12.5$ ). He was treated successfully with oral carbimazole, digoxin, and diazepam. Puerperal maternal serum concentrations of long-acting thyroid stimulator (LATS) and LATS protector (LATSP) were $45 \mathrm{units} / \mathrm{ml}$ and $283 \mathrm{units} / \mathrm{ml}$, respectively (fetal thyrotoxicosis invariably occurs when the total maternal serum LATS and LATSP concentrations exceed 20 units $/ \mathrm{ml}^{4}$ ).

When first seen in her second pregnancy her FTI was 11.3 (reference range $3 \cdot 2-12 \cdot 3)$ and serum triiodothyronine concentration $8.2 \mathrm{nmol} / 1$ $(5.3 \mathrm{ng} / \mathrm{ml}$ ) (upper limit of normal in pregnancy $4.0 \mathrm{nmol} / \mathrm{l}$ ). She therefore had T3 toxicosis. At 15 weeks oral propranolol $120 \mathrm{mg} /$ day was started, and at 25 weeks oral carbimazole $10 \mathrm{mg}$ eight-hourly. Her serum LATS and LATSP concentrations were 100 units $/ \mathrm{ml}$ and 340 units $/ \mathrm{ml}$ respectively. The fetal heart rate at 27 weeks was 168 beats/min (usual range 120-160). The dose of carbimazole was titrated against the fetal heart rate throughout the pregnancy (see figure). The propranolol was discontinued at 29 weeks.

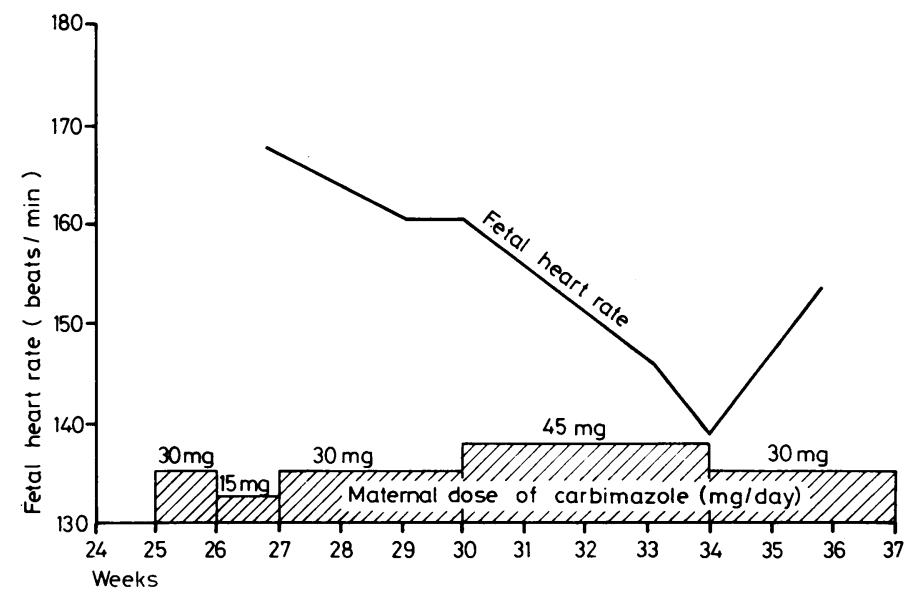

Variations of fetal heart rate related to maternal daily dose of oral carbimazole. 
At 34 weeks the mother was hypothyroid and L-thyroxine $100 \mu \mathrm{g} /$ day was started and the carbimazole was reduced. Ultrasound measurement of the fetal biparietal diameters at 32 and 36 weeks indicated a normal growth rate. At 37 weeks she delivered spontaneously a boy of $2.60 \mathrm{~kg}$ (10th-25th centile for the period of gestational age). At birth the baby was clinically thyrotoxic with a pulse rate of $169 / \mathrm{min}$. His FTI was $29 \cdot 8$. Oral carbimazole $500 \mu \mathrm{g}$ sixhourly, Lugol's iodine $1 / 15 \mathrm{ml}$ eight-hourly, and phenobarbitone $7.5 \mathrm{mg}$ eighthourly were started. By six weeks he was euthyroid and having no treatment.

\section{Comment}

A maternal history of thyrotoxicosis (particularly if recurrent after thyroidectomy), the presence of pretibial myxoedema, or a history of an affected thyrotoxic neonate are usually associated with raised serum LATS and LATSP concentrations and indicate a high probability of fetal thyrotoxicosis in subsequent pregnancies.

If there is the possibility of fetal thyrotoxicosis the fetal heart rate should be counted at each antenatal visit. A rate persistently above 160 beats/min should be considered diagnostic of this condition. The treatment of fetal thyrotoxicosis should be oral carbimazole $(10 \mathrm{mg}$ eight-hourly initially) to the mother. Carbimazole crosses the placenta and will reduce the fetal production of thyroid hormone. The dose of carbimazole should be adjusted to keep the fetal heart rate between 120 and $160 / \mathrm{min}$. Thyroxine may be added to keep the mother euthyroid if necessary. Thyroxine does not readily cross the placental barrier.

Premature labour and low birth weight for gestational age are associated with fetal thyrotoxicosis. Fetal growth rate as judged by ultrasound may be regarded as an additional criterion of the fetal thyroid state.

In this case fetal growth rate was normal. The pregnancy continued until 37 weeks (four weeks longer than the first pregnancy) and the baby was clinically and biochemically less toxic than the first child.

We thank Professor D S Munro and Dr S M Dirmikis of the University of Sheffield for their advice and for measuring the serum LATS and LATSP concentrations of this patient.

${ }^{1}$ Ramsay, I, British Medical fournal, 1976, 2, 1110.

2 Maisey, N M, and Stimmler, L, Clinical Endocrinology, 1972, 1, 81.

3 Petersen, S, and Serup, J, Acta Paediatrica Scandinavica, 1977, 66, 639.

- Dirmikis, S M, and Munro, D S, British Medical fournal, 1975, 2, 665.

(Accepted 2 Fanuary 1979)

\section{Clatterbridge Hospital, Bebington, Wirral L63 4JY}

P L ROBINSON, MD, FRCP, consultant physician

N M O'MULLANE, MRCP, DCH, senior medical registrar

BRIAN ALDERMAN, MB, MRCOG, consultant obstetrician

\section{Paratyphoid A fever diagnosed from bone marrow culture after indiscriminate antibiotic treatment}

Enteric fevers are common among immigrants and travellers returning from endemic areas. ${ }^{12}$ Blood cultures are normally diagnostic, but among patients with typhoid fever, many of whom had treated themselves with antibiotics, blood culture proved negative in $60 \%$ of cases. $^{3}$ The diagnosis was confirmed in these cases by bone marrow culture. Bone marrow culture is not, however, performed routinely when typhoid fever is suspected ${ }^{4}$ and experience of this diagnostic method in patients with paratyphoid infections is limited. ${ }^{5}$ We report here a case of paratyphoid A fever partially treated with antibiotics in which bone marrow subculture finally established the diagnosis.

\section{Case report}

A 32-year-old Pakistani woman was admitted with a three-week history of general malaise, poor appetite, weight loss, intermittent fever, abdominal pain, and diarrhoea. One week before her illness she had returned from a three-month visit to Pakistan, during which her 3-year-old son had developed a suspected Salmonella infection. She had received smallpox and cholera vaccination two weeks before travelling abroad but no $T A B$ vaccine or prophylactic antimalarial agents. Before admission she had been treated with separate four-day courses of cephalexin, amoxycillin, and chloroquine, with no clinical improvement. She denied respiratory and joint symptoms and had not noticed a rash.

She appeared unwell with temperature $38^{\circ} \mathrm{C}$, pulse rate of 90 beats $/ \mathrm{min}$, and tender hepatosplenomegaly. There was no rash and the chest was clear. Investigations showed haemoglobin $14.0 \mathrm{~g} / \mathrm{dl}$; white cell count $5 \cdot 5 \cdot 10^{9} / 1$, neutrophils $50{ }^{\circ}$, lymphocytes $40^{\circ}$, monocytes $10 \%$; erythrocyte sedimentation rate $31 \mathrm{~mm}$ in first hour. The sickle cell test was negative, as were three blood smears for malarial parasites. Two cultures each of blood and urine and single stool and liver biopsy cultures were negative. Sternal bone marrow aspirated on the third day of admission was negative on direct culture but grew paratyphoid A phage type 1 five days later after subculture from $0.1 \%$ glucose broth. The organism was sensitive to ampicillin, chloramphenicol, co-trimoxazole, tetracycline, and cephalosporins. Widal serology showed an $\mathrm{O}$ antigen titre of $1 / 30$ and an $\mathrm{H}$ titre of $1 / 320$ on admission ( $\mathrm{O}$ titres for Salmonella typhi and paratyphi $B$ were less than $1 / 20$ and $H$ titres were, respectively, $1 / 80$ and $1 / 20$ ). Two weeks later, shortly before discharge, the $\mathrm{O}$ titres for all three organisms were unchanged, the $\mathrm{H}$ titres being $1 / 640 \mathrm{for}$ paratyphi A, 1/80 for Salmonella typhi, and 1/20 for paratyphi B. Live function tests showed a raised alkaline phosphatase concentration of $48 \mathrm{Ui}$ (normal range 5-35 U/1) and aspartate transaminase concentration of $62 \mathrm{U}$ (normal range 5-40 U/1). The liver biopsy specimen showed non-specific reactive hepatitis. Bone marrow microscopy and chest radiography showed nothing abnormal.

The patient was treated with chloramphenicol $500 \mathrm{mg}$ four times a day for two weeks with ampicillin $250 \mathrm{mg}$ four times a day for the first four days only. Her fever resolved within 24 hours of starting treatment, and her symptoms rapidly settled (see figure).

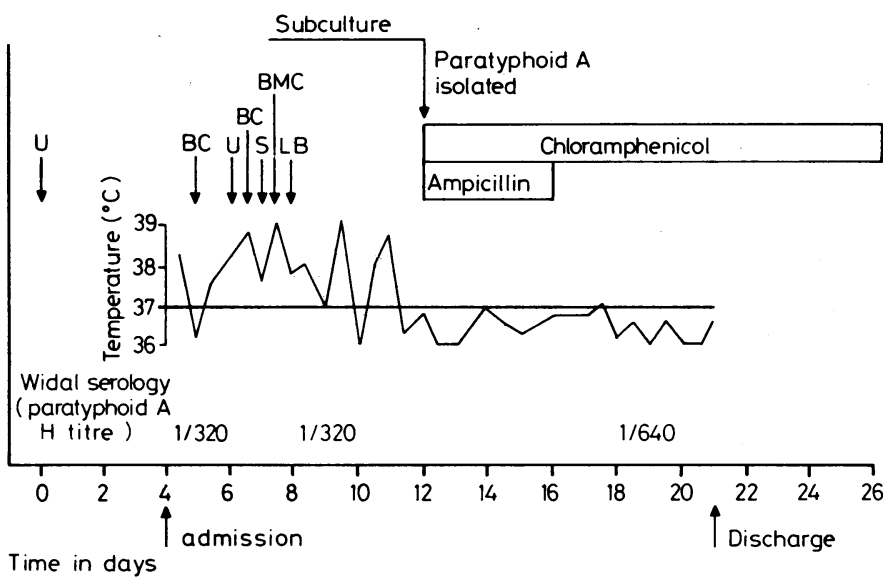

Course of disease showing changes in temperature, times of cultures, Wida serology, and response to treatment. $\mathrm{U}=$ Urine culture. $\mathrm{BC}=$ Blood culture. $\mathrm{S}=$ Stool culture. $\mathrm{BMC}=$ Bone marrow culture. $\mathrm{LB}=$ Liver biopsy culture.

\section{Comment}

This case clearly illustrates the hazards of indiscriminate antibiotic treatment in patients returning with symptoms from areas where enteric fever is endemic. Without bone marrow subculture we might not have been able to confirm the diagnosis of paratyphoid $\mathrm{A}$ fever in this patient. We suggest that bone marrow culture should become routine in all patients with suspected typhoid or paratyphoid fever who have had antibiotic treatment or when blood, urine, and stool culture results prove negative. The factors permitting survival of the organism in bone marrow remain undetermined and deserve further study.

We thank Dr I W Glick for permission to report details of this patient and Christine Bridges for secretarial help.

${ }^{1}$ British Medical fournal, 1978, 1, 377.

2 British Medical fournal, 1978, 2, 63.

${ }^{3}$ Gilman, R H, et al, Lancet, 1975, 1, 1211.

${ }^{4}$ Nye, F J, and Bell, D R, Medicine (3rd series), 1978, May, p 234.

5 Wahab, M F A, et al, Annals of Internal Medicine, 1969, 70, 913.

(Accepted 2 Fanuary 1979)

Department of Medicine, Whipps Cross Hospital, London E11

G K KNOWLES, MB, MRCP, senior registrar

M F G MURPHY, MB, BCHIR, house physician (present address: Whittington Hospital, London N19) 